\title{
BMJ Open Randomised controlled trial testing the feasibility of an exercise and nutrition intervention for patients with ovarian cancer during and after first-line chemotherapy (BENITA-study)
}

\author{
Tabea Maurer, ${ }^{1}$ Matthias Hans Belau (D) , ${ }^{2}$ Julia von Grundherr, ${ }^{3}$ Zoe Schlemmer, ${ }^{4}$ \\ Stefan Patra, ${ }^{5}$ Heiko Becher (10) ,2 Karl-Heinz Schulz, ${ }^{5,6}$ Birgit-Christiane Zyriax, ${ }^{7}$ \\ Barbara Schmalfeldt, ${ }^{4}$ Jenny Chang-Claude (1) ${ }^{1,8}$
}

To cite: Maurer T, Belau MH, von Grundherr J, et al. Randomised controlled trial testing the feasibility of an exercise and nutrition intervention for patients with ovarian cancer during and after first-line chemotherapy (BENITA-study). BMJ Open 2022;12:e054091. doi:10.1136/ bmjopen-2021-054091

- Prepublication history for this paper is available online To view these files, please visit the journal online (http://dx.doi. org/10.1136/bmjopen-2021 054091).

TM and MHB are joint first authors.

Received 09 June 2021 Accepted 24 December 2021

Check for updates

(C) Author(s) (or their employer(s)) 2022. Re-use permitted under CC BY-NC. No commercial re-use. See rights and permissions. Published by BMJ.

For numbered affiliations see end of article.

Correspondence to

Professor Jenny Chang-Claude; j.chang-claude@uke.de

\section{ABSTRACT}

Objectives Advanced ovarian cancer is a severe disease with major side effects caused by peritoneal carcinomatosis, ascites and gastrointestinal involvement as well as exhaustive treatment like debulking surgery and combination chemotherapy. Two most frequently reported side effects are muscle wasting and malnutrition, leading to frailty, decreased health-related quality of life (HRQoL) and cancer-related fatigue (CRF). As muscle wasting and malnutrition often commence during firstline chemotherapy and develop progressively into a refractory state, an early intervention is warranted. This pilot study aimed to evaluate the safety and acceptance of a combined exercise and nutrition intervention during and after first-line chemotherapy.

Design The pilot study was conducted as a monocentric 1:1 randomised controlled trial (RCT) with an intervention group (IG) and a control group (CG). Participants were divided by chance into IG or CG. Information on group allocation was conveyed to the study coordinator responsible for making an appointment with the patients for the baseline assessment as well as the physiotherapist and nutritionist responsible for the intervention and outcome assessment in both groups.

Participants Eligibility criteria included women $\geq 18$ years of age, diagnosed with ovarian cancer, tubal cancer or peritoneal cancer and primary or interval debulking, scheduled but not started adjuvant or neoadjuvant chemotherapy and sufficient German-language skills. Intervention The IG received a 12-month exercise and nutrition programme, the CG continued to follow usual care.

Primary and secondary outcome measures Primary outcomes were recruitment rate, adherence to intervention, completion rate and adverse events. In addition, in-person assessments (eg, HRQoL, CRF, muscle quality and function and dietary intake and quality) were conducted at baseline (T0, before chemotherapy), week 9 (T1, mid-chemotherapy), week 19 (T2, after completion of chemotherapy) and after 12 months of intervention (T3).

Results 0 f 60 eligible patients, 15 patients signed informed consent (recruitment rate $=25.0 \%$ ) and were
Strengths and limitations of this study

- The trial uses objective measures to evaluate the feasibility of an exercise and nutrition intervention in patients with ovarian cancer.

- The exercise and nutrition intervention commences during first-line chemotherapy and continues well into ovarian cancer survivorship.

- The exercise and nutrition intervention has been developed by an interdisciplinary team of sport and nutrition experts.

- Sport and nutrition experts conducting the intervention and assessing the outcome in both groups could not be blinded due to the study design.

randomised into IG $(n=8)$ and $C G(n=7)$. Eleven participants completed the study (completion rate, $73.3 \%$ ), one patient dropped out due to loss of interest, one due to poor health, one was lost to follow-up and one patient died.

Conclusion The BENITA (Bewegungs- und

Ernährungsintervention bei Ovarialkrebs) study demonstrated the safety and acceptance of an exercise and nutrition intervention integrated into first-line therapy and follow-up care of ovarian cancer. A large multicentre RCT is planned to investigate the effectiveness of the intervention on HRQoL, CRF and survival and to establish means of implementation into oncology guidelines and clinic routine.

Trial registration number DRKS00013231.

\section{INTRODUCTION}

Ovarian cancer is the second most common gynecologic cancer in women and has the fifth highest rate of cancer-related deaths for women in Germany ${ }^{1}$ with only $43 \%$ alive 5 years after diagnosis. ${ }^{2}$ Major side effects of ovarian cancer and its treatment are cancer cachexia, sarcopenia, frailty and malnutrition. All are leading to either loss of skeletal muscle mass and/or fat mass of the patient 
and are associated with decreased health-related quality of life (HRQoL), cancer-related fatigue (CRF) and poorer outcome. ${ }^{34}$ As these syndromes share similar aetiological factors such as reduced food intake, inflammation, hormonal changes, increased energy requirements and reduced physical activity (PA), ${ }^{5}$ more than one can be present in the same patient at the same time. Hence, a combined intervention consisting of an exercise and nutrition programme may be most successful to address these syndromes in patients with advanced cancer. ${ }^{67}$ Exercise has been shown to significantly improve CRF, cardiorespiratory fitness, HRQoL and even survival in breast and colon cancer. ${ }^{89}$ Adherence to lifestyle recommendations such as PA and nutrition before diagnosis was associated with a significantly higher HRQoL ${ }^{10}$ and decreased risk of cancers. ${ }^{11}$ However, there is paucity of knowledge on postdiagnosis PA or nutrition behaviour on prognosis or HRQoL in patients with ovarian cancer. In observational studies, patients with ovarian cancer with greater postdiagnosis PA were found to experience a significantly better HRQoL. ${ }^{12-14}$ Yet, randomised controlled trials (RCTs) evaluating the benefits of an exercise and nutrition intervention on survival and HRQoL are rare. Two RCTs on bimodal exercise and nutrition programmes for patients with ovarian cancer are currently ongoing. ${ }^{15} 16$ One commences intervention after completion of treatment ${ }^{15}$ and one investigates the effect of an intervention during first-line chemotherapy. ${ }^{16}$ However, no current or previous RCT offers a care programme during and after first-line chemotherapy, which is necessary to prevent deterioration due to treatment as well as support maintenance of lifestyle changes thereafter.

It was the aim of this study to determine the feasibility of a combined exercise and nutrition intervention for patients with ovarian cancer during and after first-line chemotherapy. Main endpoints of the pilot trial were recruitment rate, adherence, completion rate as well as adverse events (safety). Furthermore, assessments requiring visits to the hospital (in-person assessments) as planned for a main trial were conducted (eg, HRQoL, CRF, muscular strength and quality, nutrition habits and quality) to investigate acceptance and safety in patients with ovarian cancer.

\section{METHODS}

\section{Study design, setting and participants}

This pilot study was a monocentric 1:1 RCT with an intervention (IG) and a control group (CG). The ethics committee of the Faculty of Medicine at Hamburg University approved the study protocol. The trial was registered at the German Study Registry for Clinical Studies (DRKS00013231). Participants were recruited from the Department of Gynecology at the University Medical Center Hamburg-Eppendorf (UKE) in Germany at diagnosis. Eligibility criteria included women $\geq 18$ years of age, diagnosed with ovarian cancer, tubal cancer or peritoneal cancer and primary or interval debulking, scheduled but not started adjuvant or neoadjuvant chemotherapy and sufficient German-language skills. Exclusion criteria were an Eastern Cooperative Oncology Group status of two or worse, any physical or mental condition that would hinder execution or completion of the training programme and study procedures, a private engagement in exercise training above the WHO recommendation of $150 \mathrm{~min}$ of moderate-intensity activity per week ${ }^{17}$ or a diagnosis of an eating disorder.

\section{Patient and public involvement}

The patient with organisation in Germany (Verein Eierstockkrebs Deutschland e.V.) represented by its first chairperson, Andrea Krull, has provided input to the project from a patient's perspective, reviewed ethical issues and commented on consent forms.

\section{Procedure}

Two gynaecologists identified and approached participants meeting inclusion criteria. After written informed consent, patients were randomised into the IG to receive a 12-months exercise and nutrition programme or the CG to receive usual care. Group allocation was performed by a statistician not involved in data collection. Information on group allocation was conveyed to the study coordinator responsible for making an appointment with the patients for the baseline assessment as well as the physiotherapist and nutritionist responsible for the intervention, and outcome assessment in both groups.

In-person assessments were conducted independent of study arm at baseline (T0), mid-chemotherapy (T1), after completion of chemotherapy (T2), and at 1-year follow-up (T3). Assessments include HRQoL (European Organisation for Research and Treatment of Cancer (EORTC)-QLQ-C30, ${ }^{16}$ CRF (Multidimensional Fatigue Inventory (MFI-20)) ${ }^{18}$ nutritional risk (Nutritional Risk Score-2002), ${ }^{19}$ PA (Short Questionnaire to Assess Health enhancing physical activity), ${ }^{20}$ performance diagnostics including 6 min walk test, ${ }^{21}$ hand grip strength (hand grip dynamometer, 'Kern MAP 80k1'), ${ }^{22}$ accelerometer ('Actigraph wGT3X-BT') and body composition (bioelectric impedance analysis (BIA), 'AKERN BIA 101 Anniversary'). ${ }^{23} \mathrm{~A}$ detailed overview on scheduled in-person assessments is described elsewhere. ${ }^{24}$ Safety of the programme was analysed through adverse events linked to the intervention during all phases of the study. All analyses were performed using STATA MP, version V.17.

\section{Intervention}

Participants received personalised exercise and nutrition programmes and counselling that were tailored to different phases of patient's treatment and recovery as well as individual needs throughout the trial. In both phases of the exercise intervention, patients are given instructions and encouraged to participate in a daily $15-30 \mathrm{~min}$ unsupervised home-based training that includes endurance, resistance and balance exercises to be performed in gradual increments. An exercise catalogue was developed 
by sports scientists and all exercises were categorised based on their intensity. Each patient received an individually adapted programme consisting of exercises that are part of the catalogue. The programme was adjusted each week (phase I) or every other week (phase II) if needed based on the patients' individual abilities and current needs. Exercises using abdominal muscles were not included till full recovery from surgery. The exercise catalogue used to build the exercise programmes can be found in the supplements. The nutrition intervention in phase I aimed to reduce malnutrition risk by increasing protein and calorie intake. During chemotherapy, patients were supervised by a nutritionist every 3 weeks. Those who were in need of an increased calorie and protein intake were advised to consume several smaller meals throughout the day and, if necessary, to increase the use of oils and butter. Furthermore, patients were educated about suitable types of foods and drinks that are high in protein, fat or energy. If deemed necessary, oral sip feeding was suggested. These recommendations were based on the patients' development in weight as well as other body composition parameters derived from BIA measurements (eg, phase angle, muscle mass). In phase II (weeks 19-52) after chemotherapy, monthly nutrition counselling was focused on the Mediterranean diet, shown to reduce malnutrition and cancer risk. To monitor adherence and progress in phase I, participants received a weekly telephone call by a sports scientist, and triweekly by a nutritionist. In phase II, patients received monthly counselling by telephone or in person. The intervention is described in more detail elsewhere. ${ }^{24}$

\section{Statistical methods}

Recruitment rate was defined as the ratio of patients eligible to participate and patients who signed informed consent. Completion rate was defined as the ratio of patients who signed informed consent and those who completed the 12-month intervention. General adherence to the intervention was defined as the ratio of planned and completed counselling sessions. Adherence to the exercise programme was further assessed using exercise diaries filled out every week until week 18 and once a months until 12 months follow-up. Adherence to the nutrition intervention in phase I was described in terms of changes in protein and caloric intake compared with baseline. During phase II, adherence to the nutrition intervention was interpreted in terms of changes in MEDAS (Mediterranean Diet Adherence Screener) score points between T0 and T3. ${ }^{25}$ Descriptive analyses were conducted for all parameters assessed during the study. No inferential statistics were used as this feasibility trial was not powered for this purpose.

\section{RESULTS}

Characteristics and feasibility

Of 67 patients with initial diagnosis of ovarian cancer from April 2018 to Sept 2019 screened for eligibility, 60 patients met inclusion criteria and were invited into the study. 45 refused to participate in the study. Main reasons were personal reasons, residence outside of Hamburg, not willing to be randomised and no interest in the research. Fifteen patients signed informed consent (recruitment rate, $25.0 \%)$ and were randomised into IG $(n=8)$ and CG $(\mathrm{n}=7)$. Eleven participants completed the study (completion rate, $73.3 \%$ ), one patient dropped out due to loss of interest, one patient due to poor health (recurrence), one patient was lost to follow-up (could not be reached via phone or mail) and one patient died. Figure 1 provides the flow of participants through the study.

Table 1 summarises the baseline characteristics of participants by group assignment. The mean age of the participants was $56.5 \pm 14.4$ years ranging from 21 to 77 years, with an average of $33.9 \pm 17.0$ days since initial diagnosis. The majority $(73.3 \%)$ of patients was diagnosed as having advanced stage disease (stage III or IV). After surgery, eight patients had no residual tumour, five patients' tumours were resected to smaller than $1 \mathrm{~cm}$ and two patients' tumours had residual tumour larger than 1 $\mathrm{cm}$.

All 15 participants enrolled in the study completed $\mathrm{T} 0$ and $\mathrm{T} 1$ assessments. Between $\mathrm{T} 1$ and T2, one patient in the IG died and another dropped out due to loss of interest. The remaining 13 patients completed the T1 assessment. Between T2 and T3, a patient of the IG was lost to follow-up and a patient of the CG dropped out due to a recurrence. All 11 patients still enrolled in the study completed the final assessment. Table 2 provides detailed information on adherence to different assessments and time points by group assignment. Adherence to the exercise intervention in terms of completed intervention sessions (face-to-face, by telephone) was $83.7 \%$ for exercise intervention (phase I, 83.2\%; phase II, $85.1 \%$ ) and $76.8 \%$ for nutrition intervention (phase I, 92.3\%; phase II, $59.6 \%)$.

Adherence to the exercise and nutrition programme is shown in table 3. During phase I, five out of eight patients documented their weekly home-based exercise for a total of 14-18 weeks. One patient documented their daily home-based exercise for 10 weeks, another patient dropped out after 6 weeks and one patient died during phase I without documentation of home-based training. Patients trained between $90 \mathrm{~min} /$ week and 180 $\mathrm{min} /$ week. In phase II, three patients documented their exercise for 30-34 weeks. Two patients stopped their documentation after 12 and 4 weeks, respectively. Two patients dropped out of the study and one patient did not continue to document their daily practice, but remained in the study. In phase II, most patients trained for up to $90 \mathrm{~min} /$ week.

Adherence to the nutrition intervention in terms of caloric and protein intake showed that patients of the IG increased their protein intake from $65.8 \mathrm{~g}$ /day at baseline (T0) to $107.9 \mathrm{~g} /$ day at T2. The calorie intake increased from $1860 \mathrm{kcal} /$ day at T0 to $2389 \mathrm{kcal} /$ day at T2. In phase II, adherence to the nutrition intervention based on the 


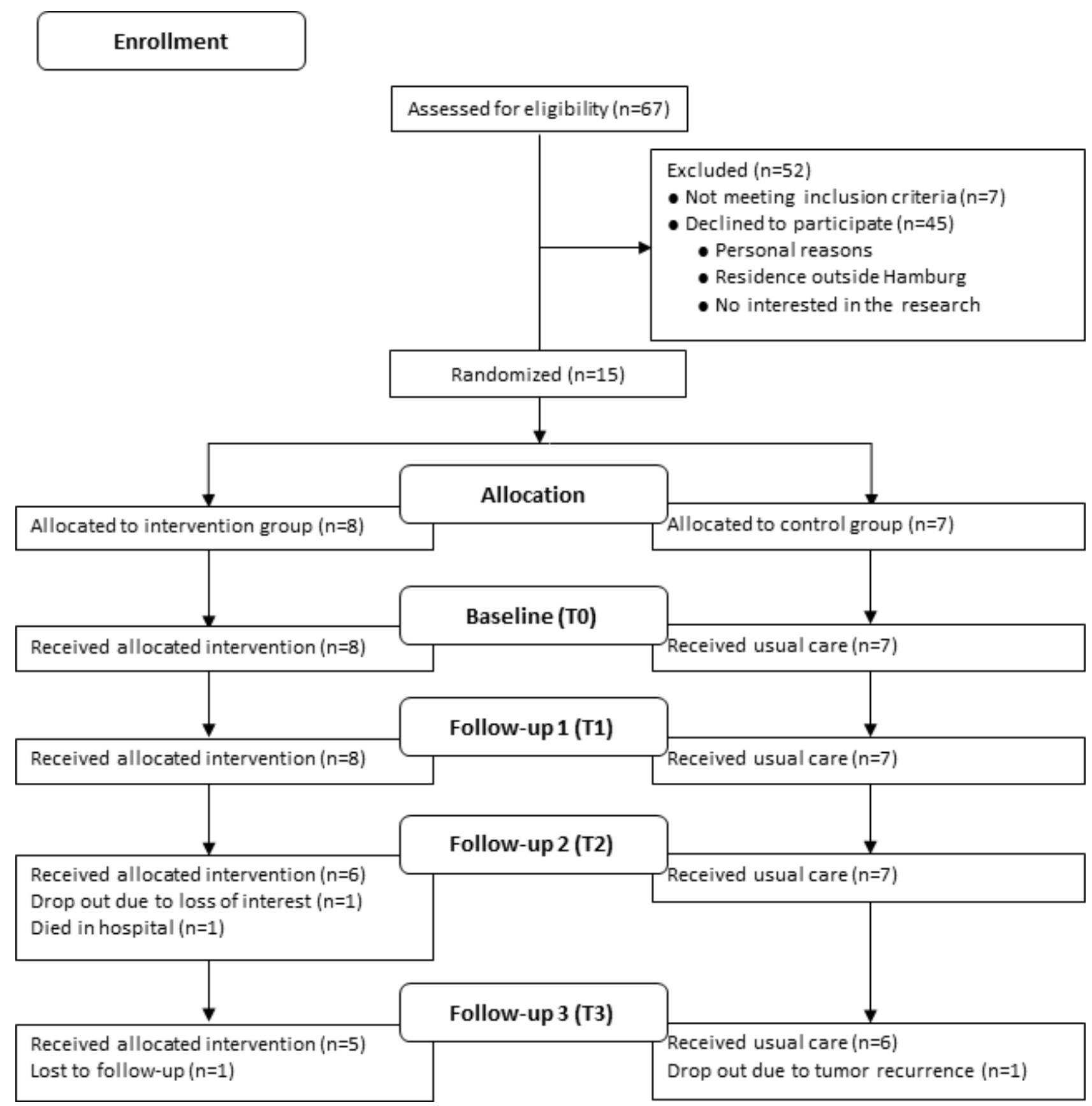

Figure 1 Flow diagram of participant recruitment and randomisation.

MEDAS score showed that patients of the IG increased their MEDAS scores from a median of 7.0 at baseline to a median of 10 score points at week 52 (T3).

Safety of the intervention was defined through any adverse events that could be linked to either the exercise or the nutrition intervention. There were no adverse events reported to be due to the intervention or in-person assessments.

\section{Descriptive statistics of in-person assessments}

Table 4 and figures 2 and 3 display descriptive results of in-person assessments at different time points by group assignment. Participants who received personalised exercise and nutrition programmes increased their median 6 min walk distance from $411 \mathrm{~m}$ at baseline to $475 \mathrm{~m}$ at T3, whereas members of the CGs decreased their distance from $440 \mathrm{~m}$ to $380 \mathrm{~m}$. Patients of the IG increased their hand grip strength from $22.0 \mathrm{~kg}$ to $24.8 \mathrm{~kg}$ (median), the CG showed a slightly lower increase (from $21.8 \mathrm{~kg}$ to 22.4 $\mathrm{kg}$ ). In terms of nutrition, calorie intake during chemotherapy increased in both IG and CG. The IG showed a larger increase in protein intake from baseline to $\mathrm{T} 1$ and T2 compared with controls. Adherence to Mediterranean diet or nutritional risk was comparable in IG and CG.

The HRQoL increased from baseline to T3 from 37.5 to 70.8 score points in the IG and from 41.7 to 50.0 score points in the CG. Both total and physical fatigue 
Table 1 Baseline characteristics of participants by group assignment

\begin{tabular}{|c|c|c|c|c|c|c|c|}
\hline & \multicolumn{2}{|c|}{ All participants } & \multicolumn{2}{|c|}{ Intervention group } & \multicolumn{2}{|c|}{ Control group } \\
\hline & & $N(=15)$ & $\%$ & $\mathrm{~N}(=8)$ & $\%$ & $\mathrm{~N}(=7)$ & $\%$ \\
\hline Age & Median (range) & \multicolumn{2}{|c|}{$58(21-77)$} & \multicolumn{2}{|c|}{$52(21-64)$} & \multicolumn{2}{|c|}{$65(48-77)$} \\
\hline \multirow{2}{*}{ Education* } & Medium & 8 & 53.3 & 5 & 62.5 & 3 & 42.9 \\
\hline & High & 6 & 40.0 & 3 & 37.5 & 3 & 42.9 \\
\hline \multirow{2}{*}{ Smoking status } & Former smoker & 5 & 33.3 & 3 & 37.5 & 2 & 28.6 \\
\hline & Current smoker & 2 & 13.3 & 1 & 12.5 & 1 & 14.3 \\
\hline \multirow[t]{3}{*}{ Alcohol use per week } & $<1 \mathrm{~g}$ & 5 & 33.3 & 3 & 37.5 & 2 & 28.6 \\
\hline & $1-12 \mathrm{~g}$ & 1 & 6.7 & 0 & 0.0 & 1 & 14.3 \\
\hline & $13-24 \mathrm{~g}$ & 3 & 20.0 & 3 & 37.5 & 0 & 0.0 \\
\hline \multirow{3}{*}{ Body mass index } & Normal weight (18.5-24.9) & 9 & 60.0 & 4 & 50.0 & 5 & 71.4 \\
\hline & Overweight (25.0-29.9) & 2 & 13.3 & 1 & 12.5 & 1 & 14.3 \\
\hline & Obesity $(\geq 30.0)$ & 3 & 20.0 & 2 & 25.0 & 1 & 14.3 \\
\hline \multirow[t]{3}{*}{ Sports $†$} & 0-4 MET h/week & 9 & 60.0 & 6 & 75.0 & 3 & 42.9 \\
\hline & 5-10 MET h/week & 1 & 6.7 & 1 & 12.5 & 0 & 0.0 \\
\hline & >10 MET h/week & 5 & 33.3 & 1 & 12.5 & 4 & 57.1 \\
\hline \multirow[t]{2}{*}{ Cancer stageł } & I & 2 & 13.3 & 1 & 12.5 & 1 & 14.3 \\
\hline & II & 2 & 13.3 & 0 & 0.0 & 2 & 28.6 \\
\hline Treatment & Neo-adjuvant chemotherapy & 3 & 20.0 & 2 & 25.0 & 1 & 14.3 \\
\hline
\end{tabular}

${ }^{*}$ CASMIN classification ${ }^{35}$

†SQUASH questionnaire ${ }^{20}$ \#FIGO classification ${ }^{36}$

CASMIN, Comparative Analysis of Social Mobility in Industrial Nations; FIGO, International Federation of Gynecology and Obstetrics; MET, metabolic equivalent of task; SQUASH, Short Questionnaire to Assess Health enhancing physical activity.

decreased from T0 to T3 and was somewhat stronger in IG than CG for physical fatigue.

\section{DISCUSSION}

This pilot trial investigating the safety, acceptance and feasibility of a combined exercise and nutrition intervention during and after first-line chemotherapy in patients with ovarian cancer demonstrated that patients were motivated to enrol and adhere to the programme and that the exercise and nutrition intervention as early as during chemotherapy were save for this vulnerable patient group.
Patients with ovarian cancer are not only seriously ill but also undergo exhausting abdominal surgery and chemotherapy. Therefore, it is not surprising that the majority of patients with ovarian cancer report an inactive lifestyle and do not meet recommendations after diagnosis and treatment. ${ }^{26}$ Common side effects of ovarian cancer and its treatment are muscle wasting and malnourishment. Both can be targeted by nutrition and exercise programmes. ${ }^{6}$ Consequently, it can be assumed that patients with ovarian cancer may benefit from an individualised exercise and/or nutrition intervention to an even greater extent than already demonstrated in patients with breast and colon cancer. ${ }^{89}$ 
Table 2 Adherence to assessment time points

\begin{tabular}{|c|c|c|c|c|c|c|c|c|}
\hline & & & \multicolumn{2}{|c|}{ All participants } & \multicolumn{2}{|c|}{ Intervention group } & \multicolumn{2}{|c|}{ Control group } \\
\hline & & & $\mathbf{N}^{*}$ & $\%$ & $\mathbf{N}^{*}$ & $\%$ & $\mathbf{N}^{*}$ & $\%$ \\
\hline \multirow[t]{7}{*}{ Exercise assessment } & Performance diagnostics & TO† & $14 / 15$ & 93.3 & $7 / 8$ & 87.5 & $7 / 7$ & 100.0 \\
\hline & & T2 & $12 / 13$ & 92.3 & $6 / 6$ & 100.0 & $6 / 7$ & 85.7 \\
\hline & & T3 & $11 / 11$ & 100.0 & $5 / 5$ & 100.0 & $6 / 6$ & 100.0 \\
\hline & Accelerometer $\ddagger$ & TO & $13 / 15$ & 86.7 & $6 / 8$ & 75.0 & $7 / 7$ & 100.0 \\
\hline & & T1 & $11 / 15$ & 73.3 & $6 / 8$ & 75.0 & $5 / 7$ & 71.4 \\
\hline & & T2 & $10 / 13$ & 76.9 & $5 / 6$ & 83.3 & $5 / 7$ & 71.4 \\
\hline & & T3 & $11 / 11$ & 100.0 & $5 / 5$ & 100.0 & $6 / 6$ & 100.0 \\
\hline \multirow{4}{*}{ Nutrition diagnostics } & & T1 & $15 / 15$ & 100.0 & $8 / 8$ & 100.0 & $7 / 7$ & 100.0 \\
\hline & & T2 & $12 / 13$ & 92.3 & $6 / 6$ & 100.0 & $6 / 7$ & 85.7 \\
\hline & & T3 & $11 / 11$ & 100.0 & $5 / 5$ & 100.0 & $6 / 6$ & 100.0 \\
\hline & & T0 - T3 & $52 / 54$ & 96.3 & $27 / 27$ & 100.0 & $25 / 27$ & 92.6 \\
\hline \multirow[t]{5}{*}{ Case report form $\S$} & & T0 & $15 / 15$ & 100.0 & $8 / 8$ & 100.0 & $7 / 7$ & 100.0 \\
\hline & & T1 & $15 / 15$ & 100.0 & $8 / 8$ & 100.0 & $7 / 7$ & 100.0 \\
\hline & & T2 & $12 / 13$ & 92.3 & $6 / 6$ & 100.0 & $6 / 7$ & 85.7 \\
\hline & & T3 & $11 / 11$ & 100.0 & $5 / 5$ & 100.0 & $6 / 6$ & 100.0 \\
\hline & & T0 - T3 & $53 / 54$ & 98.2 & $27 / 27$ & 100.0 & $26 / 27$ & 96.3 \\
\hline
\end{tabular}

${ }^{*}$ Number of participants assessed/number eligible.

†T0 = baseline, T1=mid-chemotherapy, T2=after completion of chemotherapy, T3=1-year follow-up. $\ddagger$ Worn at home for a week at each time of assessment.

§Included all questionnaires applied In the study.

As ovarian cancer is often diagnosed at a late stage of disease and the median age at initial diagnosis is 62 years, it was anticipated that the recruitment and completion rate would be lower than that reported in studies including patients with cancer diagnosed at an early stage or at a younger age.$^{27}$ In our randomised feasibility trial, recruitment rate was $25.0 \%$, which is in line with recruitment rates of $16 \%-63 \%$ and a retention rates of $70-100$ stated in a recent review. ${ }^{14}$ Reported reasons for refusal of participation were symptoms, illness and exhaustion. ${ }^{14}$ These reasons hold true for our study as well. In addition, many patients declined to take part due to a distant residence, which was also the reason for not undergoing chemotherapy at UKE, thus requiring separate trips to UKE for the study. Others did not participate because they were not willing to risk randomisation into the CG. Patients who consented to participate in the study showed a high commitment, and only two patient(s) dropped out, leading to a completion rate of $73.3 \%$. Adherence to the exercise intervention in terms of completed counselling sessions was higher than reported by a systematic review ${ }^{14}$ with $83.7 \%$ for exercise intervention (phase I, 83.2\%; phase II, $85.1 \%$ ) and $76.8 \%$ for nutrition intervention (phase I, 92.3\%; phase II, 59.6\%). There were no adverse events associated with the intervention documented throughout the trial. Therefore, this study, to our knowledge, is the first to show that a combined nutrition and exercise intervention in patients with ovarian cancer during and after first-line chemotherapy is feasible, safe and accepted.

To date, few RCTs on exercise and/or nutrition in ovarian cancer exist and those few available mainly recruited patiens after completion of treatment. Thus, these studies in principle predominantly recruited patients in remission free of progression. The Women's Activity and Lifestyle Study in Connecticut (WALC) ${ }^{28}$ trial, a 6-month exercise intervention in ovarian cancer, for example, included patients up to 4 years following initial diagnosis, and the patients' sample was, therefore, heterogeneous. The Resistance and Endurance exercise After ChemoTherapy (REACT) study ${ }^{9}$ including a few patients with ovarian cancer among other cancer survivors used a 12-week exercise intervention without combined nutrition counselling shortly after completion of treatment. The currently ongoing Lifestyle Intervention for Ovarian Cancer Enhanced Survival (LIVES) study ${ }^{15}$ also 
Table 3 Adherence to the implementation of exercise and nutrition programme among participants from the intervention group

\section{Exercise programme}

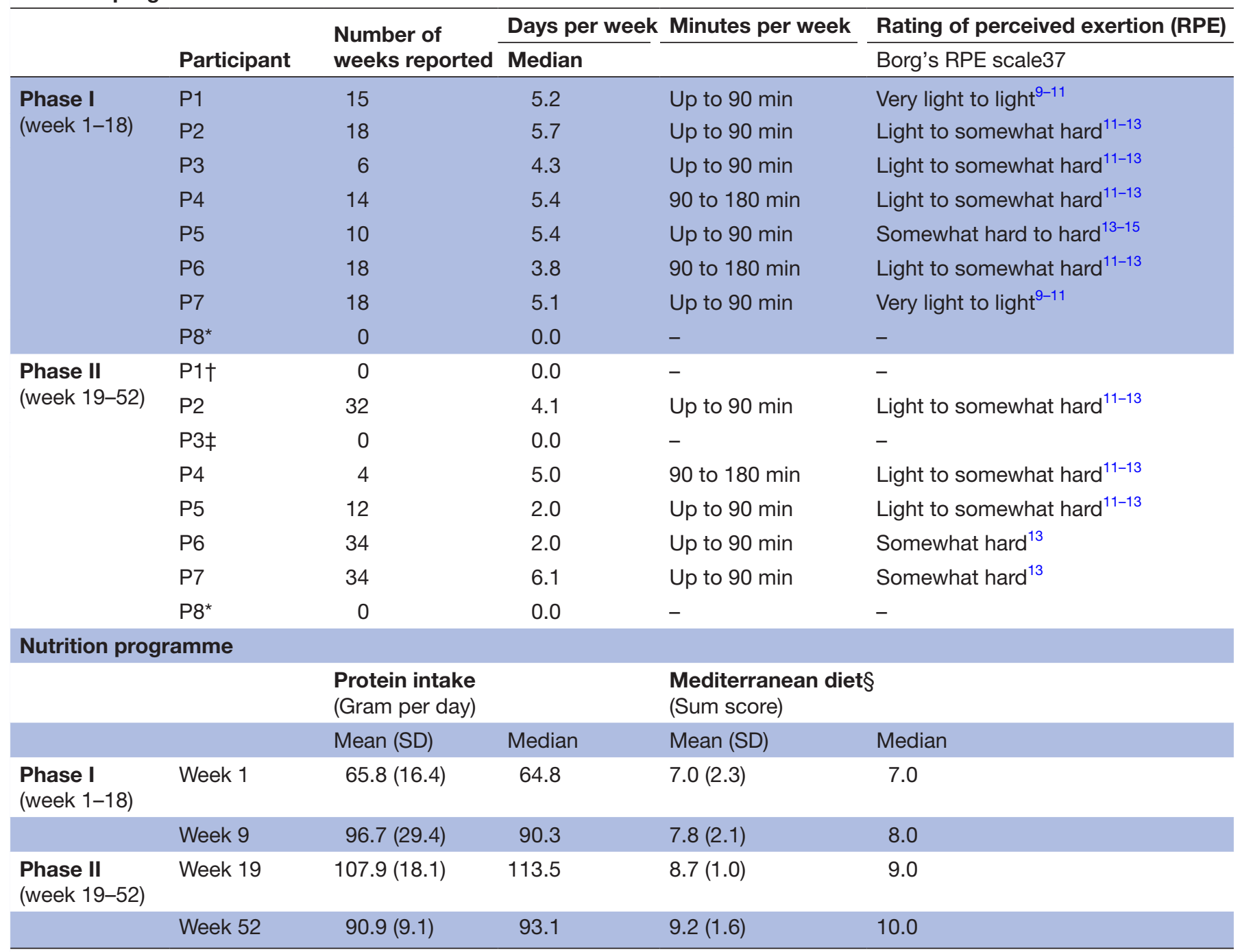

*Died in hospital.

†Dropped out.

‡Lost to follow-up.

§MEDAS sum score.

MEDAS, Mediterranean Diet Adherence Screener.

investigates the effect of a 24-month lifestyle intervention after treatment for patients with ovarian cancer. Only the ongoing Physical Activity and Dietary intervention in women with OVArian cancer (PADOVA) study offers a combined exercise and nutrition intervention during firstline chemotherapy. ${ }^{29}$ However, the exercise and nutrition intervention are limited to the duration of chemotherapy only, whereas our study aims to start with chemotherapy and to continue well into ovarian cancer survivorship to ensure maintenance of the recommended lifestyle.

Previous studies on postdiagnosis exercise in ovarian cancer have shown that exercise leads to improvements in HRQOL, fatigue and additional physical and psychological outcomes. ${ }^{14}$ The few feasibility studies on exercise and/or nutrition interventions during first-line chemotherapy reported increased moderate to strenuous PA to be correlated with improvements in HRQOL ${ }^{30-32}$ and physical functioning (eg, muscular strength, 6 min walking test $)^{30-32}$ as well as reduced fatigue. ${ }^{31} 32$ Our study showed similar tendencies for the 6 min walking test, physical fatigue as well as global health. However, these results are descriptive only and no RCT exists to prove effectiveness of a combined exercise and nutrition intervention during and/or after primary care in patients with ovarian cancer.

\section{CONCLUSION}

To date, guidelines on care programmes for patients with ovarian cancer in Germany are based solely on 
Table 4 Results of assessments at different time points by group assignment

\begin{tabular}{|c|c|c|c|c|c|c|c|}
\hline & & \multicolumn{2}{|c|}{ All participants } & \multicolumn{2}{|c|}{ Intervention group } & \multicolumn{2}{|l|}{ Control group } \\
\hline & & Mean (SD) & Median & Mean (SD) & Median & Mean (SD) & Median \\
\hline \multirow{4}{*}{$\begin{array}{l}6 \text { min walking test } \\
\text { meter }\end{array}$} & TO* & $397.5(109.8)$ & 411.0 & 369.7 (126.3) & 325.7 & $436.4(77.3)$ & 440.0 \\
\hline & $\mathbf{T 1}$ & $489.0(95.5)$ & 490.0 & $483.9(96.2)$ & 495.0 & 495.1 (105.6) & 458.8 \\
\hline & T2 & $496.2(116.5)$ & 507.7 & $511.9(80.9)$ & 524.4 & $477.4(157.8)$ & 410.0 \\
\hline & T3 & $492.8(134.6)$ & 475.0 & $542.4(91.1)$ & 570.7 & $451.5(158.4)$ & 380.0 \\
\hline \multirow{4}{*}{$\begin{array}{l}\text { Hand grip strength } \dagger \\
\text { kilogram }\end{array}$} & T0 & $22.4(7.0)$ & 21.9 & $22.2(8.7)$ & 22.0 & $22.6(4.7)$ & 21.8 \\
\hline & $\mathbf{T 1}$ & $24.1(7.5)$ & 21.6 & $23.5(6.4)$ & 21.6 & $25.0(9.9)$ & 21.3 \\
\hline & T2 & $25.2(6.9)$ & 24.6 & $23.0(6.0)$ & 23.2 & $27.8(7.6)$ & 25.8 \\
\hline & T3 & $25.6(6.7)$ & 24.8 & $26.3(5.9)$ & 24.8 & $25.1(7.8)$ & 22.4 \\
\hline \multirow{4}{*}{$\begin{array}{l}\text { Mediterranean diet } \neq \\
\text { sum core }\end{array}$} & TO & $7.0(1.9)$ & 8.0 & $7.0(2.3)$ & 7.0 & $7.0(1.4)$ & 8.0 \\
\hline & T1 & $8.4(2.2)$ & 9.0 & $7.8(2.1)$ & 8.0 & $9.1(2.2)$ & 9.0 \\
\hline & T2 & $9.0(1.8)$ & 9.0 & $8.7(1.0)$ & 9.0 & $9.3(2.3)$ & 9.5 \\
\hline & T3 & $9.2(1.9)$ & 10.0 & $9.2(1.6)$ & 10.0 & $9.2(2.3)$ & 9.0 \\
\hline \multirow{4}{*}{$\begin{array}{l}\text { Nutritional risk§ } \\
\text { sum score }\end{array}$} & T0 & $3.4(1.1)$ & 3.0 & $3.5(1.2)$ & 3.5 & $3.3(1.1)$ & 3.0 \\
\hline & $\mathbf{T 1}$ & $3.1(1.3)$ & 3.0 & $3.0(1.5)$ & 2.5 & $3.3(1.1)$ & 3.0 \\
\hline & T2 & $2.4(1.8)$ & 2.5 & $2.5(2.1)$ & 3.0 & $2.3(1.6)$ & 2.0 \\
\hline & T3 & $0.4(0.7)$ & 0.0 & $0.2(0.5)$ & 0.0 & $0.5(0.8)$ & 0.0 \\
\hline \multirow{4}{*}{$\begin{array}{l}\text { Protein intake } \\
\text { gram per day }\end{array}$} & TO & $68.0(13.3)$ & 64.8 & $65.8(16.4)$ & 64.8 & $70.6(9.1)$ & 68.1 \\
\hline & $\mathbf{T 1}$ & $89.6(30.4)$ & 87.0 & $96.7(29.4)$ & 90.3 & $78.2(31.4)$ & 79.6 \\
\hline & T2 & $104.0(23.5)$ & 113.3 & $107.9(18.1)$ & 113.5 & $100.1(29.1)$ & 97.3 \\
\hline & T3 & $89.3(23.0)$ & 93.1 & $90.9(9.1)$ & 93.1 & $87.9(31.4)$ & 93.6 \\
\hline \multirow{4}{*}{$\begin{array}{l}\text { Caloric intake } \\
\text { kilocalories per day }\end{array}$} & T0 & $1830(382)$ & 1816 & $1860(388)$ & 1987 & 1795 (409) & 1663 \\
\hline & $\mathbf{T 1}$ & $2237(612)$ & 2439 & $2380(429)$ & 2350 & $2010(835)$ & 2439 \\
\hline & T2 & $2237(513)$ & 2439 & 2389 (372) & 2474 & 2147 (635) & 2071 \\
\hline & T3 & $2206(548)$ & 2355 & 2105 (398) & 2219 & 2291 (675) & 2387 \\
\hline \multicolumn{8}{|l|}{ HRQoLף } \\
\hline \multirow{4}{*}{$\begin{array}{l}\text { Global health status } \\
\text { sum score }\end{array}$} & TO & $40.0(10.5)$ & 41.7 & $40.6(8.3)$ & 37.5 & $39.3(13.4)$ & 41.7 \\
\hline & T1 & $55.6(27.8)$ & 66.7 & $62.5(20.4)$ & 66.7 & $47.6(34.3)$ & 33.3 \\
\hline & T2 & $59.7(20.7)$ & 54.2 & $58.3(14.9)$ & 54.2 & $61.1(26.7)$ & 54.2 \\
\hline & T3 & $65.8(19.8)$ & 66.7 & $72.9(8.0)$ & 70.8 & $61.1(24.5)$ & 50.0 \\
\hline \multirow{4}{*}{$\begin{array}{l}\text { Physical functioning } \\
\text { sum score }\end{array}$} & TO & $59.1(25.1)$ & 66.7 & $54.2(27.5)$ & 53.3 & $64.8(22.7)$ & 66.7 \\
\hline & T1 & $69.3(23.1)$ & 73.3 & 66.7 (23.9) & 76.7 & $72.4(23.5)$ & 73.3 \\
\hline & T2 & $70.6(21.9)$ & 76.7 & 76.7 (12.5) & 80.0 & $64.4(28.5)$ & 63.3 \\
\hline & T3 & $78.2(16.9)$ & 73.3 & $76.0(17.4)$ & 73.3 & $80.0(17.9)$ & 76.7 \\
\hline \multicolumn{8}{|l|}{$\mathrm{CRF}^{\star \star}$} \\
\hline \multirow{4}{*}{$\begin{array}{l}\text { General fatigue } \\
\text { sum score }\end{array}$} & TO & $17.6(5.3)$ & 18.0 & $18.6(5.2)$ & 17.5 & $16.6(5.7)$ & 18.0 \\
\hline & T1 & $14.9(6.3)$ & 14.0 & $13.9(5.1)$ & 14.0 & $16.1(7.7)$ & 14.0 \\
\hline & T2 & $14.5(6.2)$ & 15.0 & $15.2(6.1)$ & 15.0 & $13.8(7.0)$ & 13.0 \\
\hline & T3 & $12.8(6.2)$ & 12.0 & $13.8(7.2)$ & 11.0 & $11.8(5.6)$ & 13.0 \\
\hline \multirow{4}{*}{$\begin{array}{l}\text { Physical fatigue } \\
\text { sum score }\end{array}$} & T0 & $18.5(6.0)$ & 17.0 & $19.1(6.7)$ & 18.5 & $17.7(5.5)$ & 17.0 \\
\hline & T1 & $14.0(7.1)$ & 15.0 & $12.3(7.4)$ & 9.5 & $16.0(6.6)$ & 17.0 \\
\hline & T2 & $12.9(5.8)$ & 12.0 & $12.0(4.9)$ & 11.0 & $14.0(7.3)$ & 16.0 \\
\hline & T3 & $11.6(5.9)$ & 9.5 & $11.0(6.4)$ & 7.0 & $12.2(5.9)$ & 12.0 \\
\hline
\end{tabular}

${ }^{*} \mathrm{~T} 0$ = baseline, $\mathrm{T} 1=$ mid-chemotherapy, $\mathrm{T} 2=$ after completion of chemotherapy, T3=1-year FU.

tdominant hand.

†MEDAS.

§NRS-2002.

ПEORTC QLQ-C30.

${ }^{* *} \mathrm{MFI}-20$.

CRF, cancer-related fatigue; EORTC, European Organisation for Research and Treatment of Cancer; HRQoL, health-related quality of life; MEDAS, Mediterranean Diet Adherence

Screener; MFI, Multidimensional Fatigue Inventory; NRS, Nutritional Risk Score. 
Six minute walking test Median
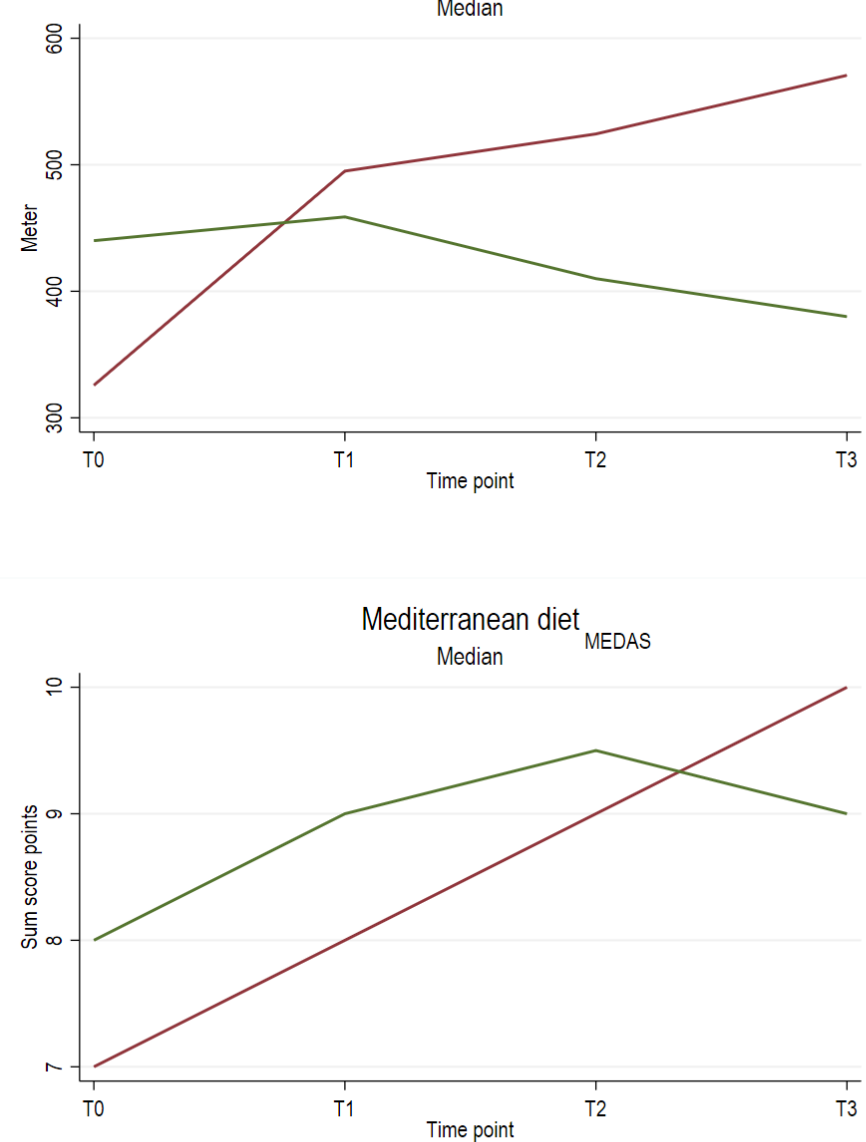

Protein intake

Median

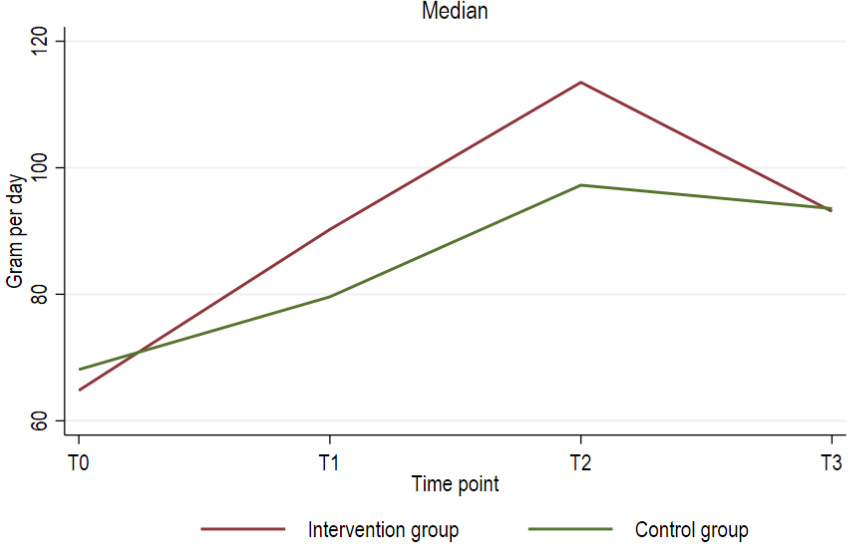

Hand grip strength

Median

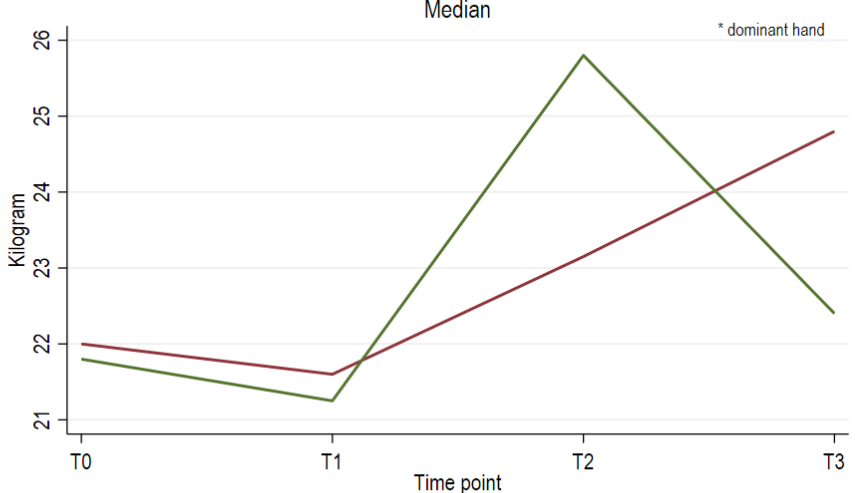

Nutritional risk Median NRS-2002

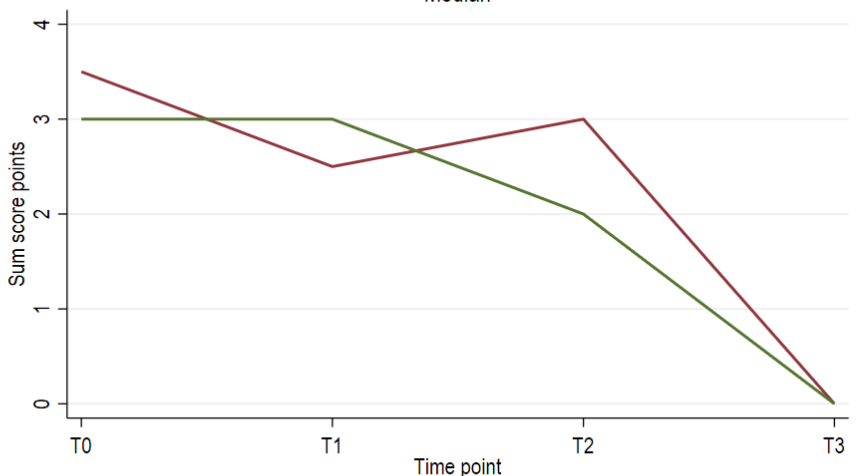

Caloric intake Median

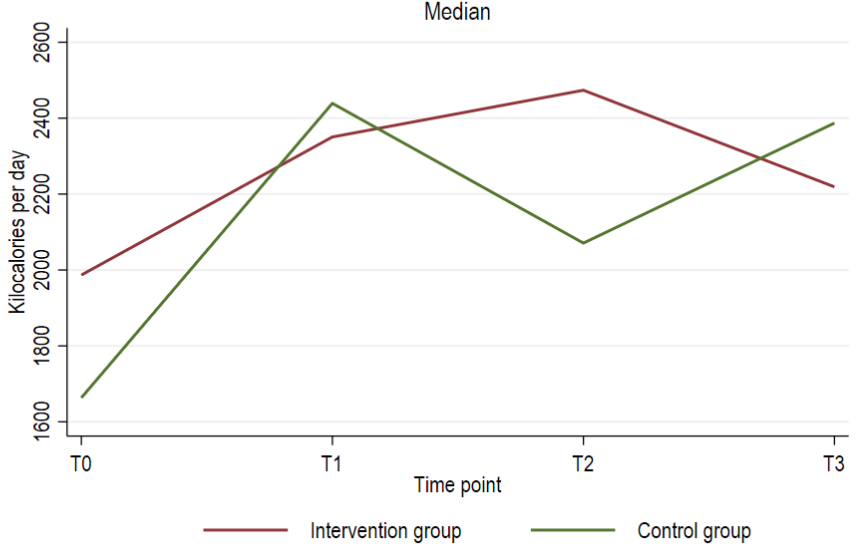

Figure 2 Descriptive results of in-person assessments at baseline (T0), mid-chemotherapy (T1), after completion of chemotherapy (T2), and 1 year follow-up (T3) by group assignment. MEDAS, Mediterranean Diet Adherence Screener.

expert consensus. ${ }^{33}$ Although aftercare programmes for ovarian cancer survivors to improve HRQoL and CRF are recommended, current treatment guidelines include a further 15-24-month maintenance therapy after completion of chemotherapy, which renders it difficult for patients to receive inpatient rehabilitation after first-line therapy. ${ }^{33}$ Therefore, of about a third of patients who survive for more than 8 years up to $70 \%$ will suffer long-term sequelae of cancer treatment, including reduced HRQoL and $\mathrm{CRF}^{34}$ A home-based personalised standardised care intervention programme beginning already during chemotherapy and continued post-treatment will enable the majority of patients to participate and further empower them to achieve 

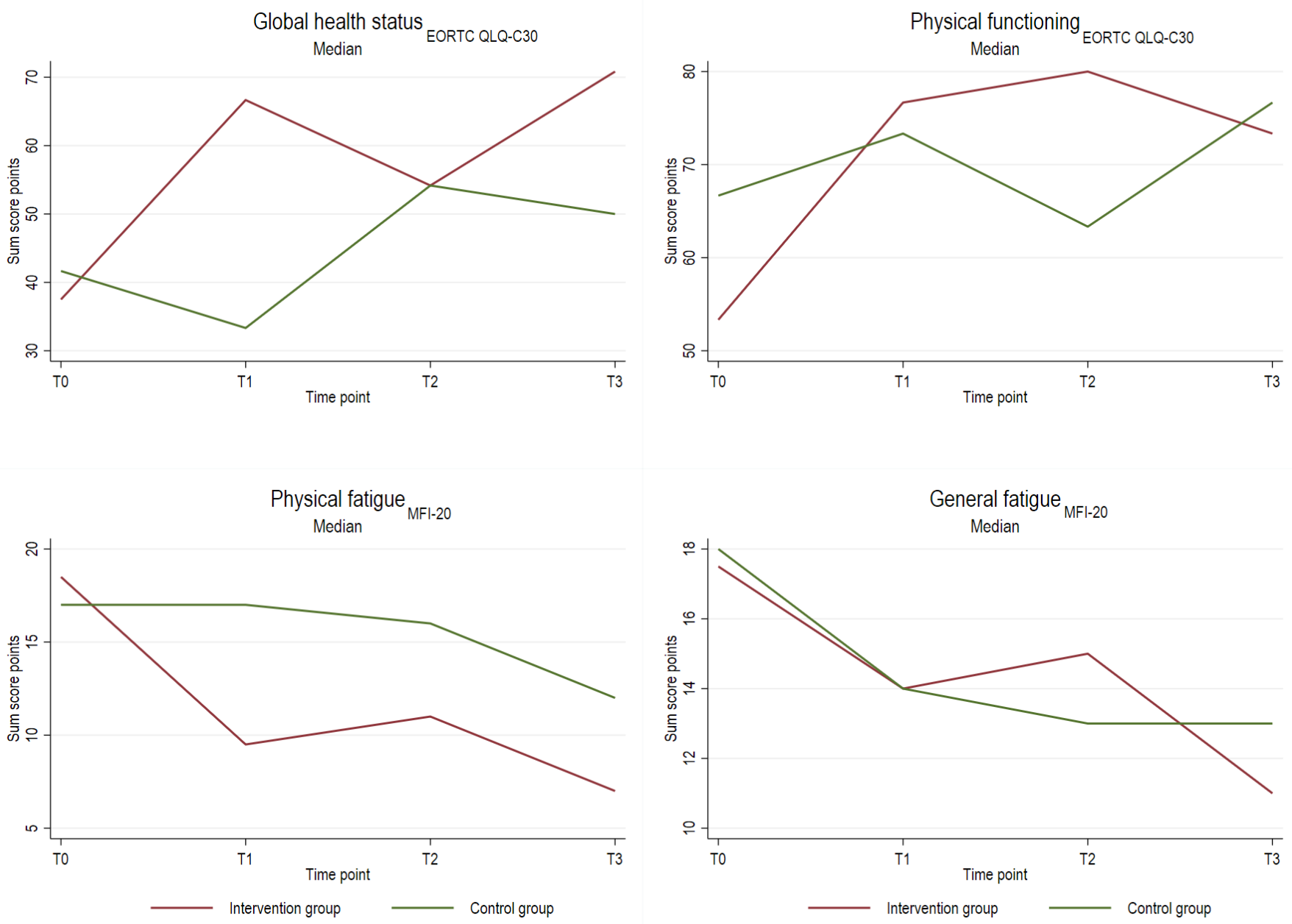

Figure 3 Descriptive results (continued) of in-person assessments at baseline (T0), mid-chemotherapy (T1), after completion of chemotherapy (T2), and 1 year follow-up (T3) by group assignment. EORTC, European Organisation for Research and Treatment of Cancer; MFI, Multidimensional Fatigue Inventory; NRS, Nutritional Risk Score.

long-term adherence to recommended exercise and nutrition behaviour.

Thus, following this pilot study, it will be important to conduct a multicentre RCT (1) to provide evidence of the effectiveness of a personalised combined exercise and nutrition intervention during adjuvant and maintenance chemotherapy compared with standard care to improve HRQoL and reduce CRF in patients with ovarian cancer and (2) to establish an exercise and nutrition programme ready for implementation into routine clinical practice for patients with ovarian cancer.

\section{Author affiliations}

${ }^{1}$ Cancer Epidemiology, University Cancer Center Hamburg (UCCH), University Medical Center Hamburg-Eppendorf, Hamburg, Germany

${ }^{2}$ Medical Biometry and Epidemiology, University Medical Center HamburgEppendorf, Hamburg, Germany

${ }^{3}$ Department of Oncology, Hematology, BMT with Section Pneumology, Hubertus Wald Tumour Center, University Cancer Center Hamburg (UCCH), University Medical Center Hamburg-Eppendorf, Hamburg, Germany

${ }^{4}$ Department of Gynaecology and Gynaecologic Oncology, University Medical Center Hamburg-Eppendorf, Hamburg, Germany

${ }^{5}$ Center for Athletic Medicine (UKE Athleticum), University Medical Center HamburgEppendorf, Hamburg, Germany
${ }^{6}$ Department of Medical Psychology, University Medical Center Hamburg-Eppendorf, Hamburg, Germany

${ }^{7}$ Midwifery Science - Health Care Research and Prevention, Institute for Health Services Research in Dermatology and Nursing (IVDP), University Medical Center Hamburg-Eppendorf, Hamburg, Germany

${ }^{8}$ Division of Cancer Epidemiology, DKFZ, Heidelberg, Baden-Württemberg, Germany

Acknowledgements We would like to thank all patients who participated in the BENITA study as well as Andrea Krull (Verein Eierstockkrebs Deutschland e.V.) for her helpful advice on the study plan. The authors would also like to thank Professor Dr S C Bischoff of the University Hohenheim for providing them with the validated German version of the MEDAS questionnaire.

Contributors TM, JvG, SP, KHS, BS, B-CZ and JC-C contributed to study conception and design. TM, MHB, JvG and ZS contributed to data and sample collection. JC-C obtained funding for the pilot project. TM, MHB and JC-C drafted the first version of the manuscript. MHB is responsible for data management of pilot study. HB performed the sample size calculations and supervised randomisation process. All authors revised the protocol critically for important intellectual content and read and approved the final version of the protocol. JC-C is guarantor.

Funding The pilot phase of the BENITA study has been funded for 2 years by the Hamburger Krebsgesellschaft e.V. (grant number: not applicable). There is no pharmaceutical industry funding and there are no commercial interests.

Competing interests None declared. 
Patient and public involvement Patients and/or the public were involved in the design, or conduct, or reporting, or dissemination plans of this research. Refer to the Methods section for further details.

\section{Patient consent for publication Not applicable.}

Ethics approval This study involves human participants and was approved by The ethics committee of the Faculty of Medicine at Hamburg University approved the study Participants gave informed consent to participate in the study before taking part.

\section{Provenance and peer review Not commissioned; externally peer reviewed.}

Data availability statement Data are available upon reasonable request. Data cannot be made publicly available for legal reasons. Due to data privacy rules and according to German law ( $§ 75$ SGB X) access to the data is granted only to responsible scientific personnel at UKE, Hamburg, Germany within the framework of the respective research project. It is not permitted to give third parties access to the data without a research proposal approved by the principal investigator.

Open access This is an open access article distributed in accordance with the Creative Commons Attribution Non Commercial (CC BY-NC 4.0) license, which permits others to distribute, remix, adapt, build upon this work non-commercially, and license their derivative works on different terms, provided the original work is properly cited, appropriate credit is given, any changes made indicated, and the use is non-commercial. See: http://creativecommons.org/licenses/by-nc/4.0/.

\section{ORCID iDs}

Matthias Hans Belau http://orcid.org/0000-0003-2141-2162

Heiko Becher http://orcid.org/0000-0002-8808-6667

Jenny Chang-Claude http://orcid.org/0000-0001-8919-1971

\section{REFERENCES}

1 Ferlay J, Steliarova-Foucher E, Lortet-Tieulent J, et al. Cancer incidence and mortality patterns in Europe: estimates for 40 countries in 2012. Eur J Cancer 2013;49:1374-403.

2 Robert Koch-Institute. Krebs in Deutschland 2015/2016. Berlin, 2019.

3 Rutten IJG, van Dijk DPJ, Kruitwagen RFPM, et al. Loss of skeletal muscle during neoadjuvant chemotherapy is related to decreased survival in ovarian cancer patients. J Cachexia Sarcopenia Muscle 2016;7:458-66.

4 Fearon KC, Barber MD, Moses AG. The cancer cachexia syndrome. Surg Oncol Clin N Am 2001;10:109-26.

5 Gingrich A, Volkert D, Kiesswetter E, et al. Prevalence and overlap of sarcopenia, frailty, cachexia and malnutrition in older medical inpatients. BMC Geriatr 2019;19:120.

6 Fearon $\mathrm{KCH}$. Cancer cachexia: developing multimodal therapy for a multidimensional problem. Eur J Cancer 2008;44:1124-32.

7 Johns DJ, Hartmann-Boyce J, Jebb SA, et al. Diet or exercise interventions vs combined behavioral weight management programs: a systematic review and meta-analysis of direct comparisons. J Acad Nutr Diet 2014;114:1557-68.

8 McNeely ML, Campbell KL, Rowe BH, et al. Effects of exercise on breast cancer patients and survivors: a systematic review and metaanalysis. CMAJ 2006;175:34-41.

9 Kampshoff CS, Chinapaw MJM, Brug J, et al. Randomized controlled trial of the effects of high intensity and low-to-moderate intensity exercise on physical fitness and fatigue in cancer survivors: results of the resistance and endurance exercise after chemotherapy (react) study. BMC Med 2015;13:275.

10 Blanchard CM, Courneya KS, Stein K, et al. Cancer survivors adherence to lifestyle behavior recommendations and associations with health-related quality of life: results from the American cancer Society's SCS-II. J Clin Oncol 2008;26:2198-204.

11 McCullough ML, Patel AV, Kushi LH, et al. Following cancer prevention guidelines reduces risk of cancer, cardiovascular disease, and all-cause mortality. Cancer Epidemiol Biomarkers Prev 2011;20:1089-97.

12 Smits A, Smits E, Lopes A, et al. Body mass index, physical activity and quality of life of ovarian cancer survivors: time to get moving? Gynecol Oncol 2015;139:148-54.

13 Stevinson C, Faught W, Steed H, et al. Associations between physical activity and quality of life in ovarian cancer survivors. Gynecol Oncol 2007;106:244-50.

14 Jones TL, Sandler CX, Spence RR, et al. Physical activity and exercise in women with ovarian cancer: a systematic review. Gynecol Oncol 2020;158:803-11.
15 Thomson CA, Crane TE, Miller A, et al. A randomized trial of diet and physical activity in women treated for stage II-IV ovarian cancer: rationale and design of the lifestyle intervention for ovarian cancer enhanced survival (lives): an NRG Oncology/Gynecologic Oncology Group (GOG-225) study. Contemp Clin Trials 2016;49:181-9.

16 Stelten S, Hoedjes M, Kenter GG, et al. Rationale and study protocol of the physical activity and dietary intervention in women with ovarian cancer (Padova) study: a randomised controlled trial to evaluate effectiveness of a tailored exercise and dietary intervention on body composition, physical function and fatigue in women with ovarian cancer undergoing chemotherapy. BMJ Open 2020;10:e036854.

17 Piercy KL, Troiano RP, Ballard RM, et al. The physical activity guidelines for Americans. JAMA 2018;320:2020-8.

18 Smets EM, Garssen B, Bonke B, et al. The multidimensional fatigue inventory (MFI) psychometric qualities of an instrument to assess fatigue. J Psychosom Res 1995;39:315-25.

19 Kondrup J, Rasmussen $\mathrm{HH}$, Hamberg O, et al. Nutritional risk screening (NRS 2002): a new method based on an analysis of controlled clinical trials. Clin Nutr 2003;22:321-36.

20 Wendel-Vos GCW, Schuit AJ, Saris WHM, et al. Reproducibility and relative validity of the short questionnaire to assess health-enhancing physical activity. J Clin Epidemiol 2003;56:1163-9.

21 Agarwala P, Salzman SH. Six-Minute walk test: clinical role, technique, coding, and reimbursement. Chest 2020;157:603-11.

22 Butte NF, Ekelund U, Westerterp KR. Assessing physical activity using wearable monitors: measures of physical activity. Med Sci Sports Exerc 2012;44:S5-12.

23 Cotogni $\mathrm{P}$, Monge T, Fadda M, et al. Bioelectrical impedance analysis for monitoring cancer patients receiving chemotherapy and home parenteral nutrition. BMC Cancer 2018;18:990.

24 Maurer T, von Grundherr J, Patra S, et al. An exercise and nutrition intervention for ovarian cancer patients during and after first-line chemotherapy (BENITA study): a randomized controlled pilot trial. Int J Gynecol Cancer 2020;30:541-5.

25 Hebestreit K, Yahiaoui-Doktor M, Engel C, et al. Validation of the German version of the Mediterranean diet adherence screener (MEDAS) questionnaire. BMC Cancer 2017;17:341.

26 Mizrahi D, Naumann F, Broderick C, et al. Quantifying physical activity and the associated barriers for women with ovarian cancer. Int J Gynecol Cancer 2015;25:577-83.

27 Sheill G, Guinan E, Brady L, et al. Exercise interventions for patients with advanced cancer: a systematic review of recruitment, attrition, and exercise adherence rates. Palliat Support Care 2019;17:686-96.

28 Zhou Y, Gottlieb L, Cartmel B, et al. Randomized trial of exercise on quality of life and fatigue in women diagnosed with ovarian cancer: The Women's Activity and Lifestyle Study in Connecticut (WALC). Journal of Clinical Oncology 2015;33:9505.

29 Stelten S, Hoedjes M, Kenter GG, et al. Rationale and study protocol of the physical activity and dietary intervention in women with ovarian cancer (Padova) study: a randomised controlled trial to evaluate effectiveness of a tailored exercise and dietary intervention on body composition, physical function and fatigue in women with ovarian cancer undergoing chemotherapy. BMJ Open 2020;10:e036854.

30 Newton MJ, Hayes SC, Janda M, et al. Safety, feasibility and effects of an individualised walking intervention for women undergoing chemotherapy for ovarian cancer: a pilot study. BMC Cancer 2011:11:389.

31 Mizrahi D, Broderick C, Friedlander M, et al. An exercise intervention during chemotherapy for women with recurrent ovarian cancer: a feasibility study. Int J Gynecol Cancer 2015;25:985-92.

32 von Gruenigen VE, Frasure HE, Kavanagh MB, et al. Feasibility of a lifestyle intervention for ovarian cancer patients receiving adjuvant chemotherapy. Gynecol Oncol 2011;122:328-33.

33 Wagner U, Harter P, Hilpert F, et al. S3-Guideline on Diagnostics, Therapy and Follow-up of Malignant Ovarian Tumours: Short version 1.0 - AWMF registration number: 032/035OL, June 2013. Geburtshilfe Frauenheilkd 2013;73:874-89.

34 Joly F, Ahmed-Lecheheb D, Kalbacher E, et al. Long-Term fatigue and quality of life among epithelial ovarian cancer survivors: a GINECO case/control VIVROVAIRE I study. Ann Oncol 2019;30:845-52

35 Brauns HSS, Steinmann S. The CASMIN educational classification in international comparative. research. Boston, MA: Springer, 2003.

36 Prat J, FIGO Committee on Gynecologic Oncology. Figo's staging classification for cancer of the ovary, fallopian tube, and peritoneum: abridged republication. J Gynecol Oncol 2015;26:87-9.

37 Borg GA. Psychophysical bases of perceived exertion. Med Sci Sports Exerc 1982;14:377???381-81. 